\title{
Frühzeitiger Einsatz senkt Intubationsrate und Letalität
}

- COPD-Patienten benötigen in fortgeschrittenen Stadien häufig eine nicht invasive Beatmung (NIV). Meist ist eine akute Exazerbation der Auslöser für den Beginn einer NIV. Laut S3-Leitlinie sollte die NIV bei milder bis mittelgradiger akut exazerbierter $\mathrm{COPD}(\mathrm{pH}-$ Wert 7,30-7,35) frühzeitig eingesetzt werden, da sie nicht nur die Atempumpe entlastet, sondern auch die Intubationsrate und die Letalität senkt. Ein Therapieversuch kann auch bei schwergradiger Azidose unternommen werden, wie Prof. Bernd Schönhofer, Oststadt-Heidehaus, ausführte.

\section{Atemarbeit sinkt vergleichbar}

Die Atemarbeit nimmt bei NIV vergleichbar ab wie bei invasiver Beatmung, vorausgesetzt, es wird ein ausreichend hoher Beatmungsdruck gewählt und es bleibt genügend Zeit für die Exspiration. Gründe doch zu intubieren sind erschöpfte Atemmuskulatur, massive Hypersekretion oder mentale Erschöpfung.
Bei COPD-Patienten, die intubiert wurden, ist die Entwöhnung (weaning) vom Respirator wegen Erschöpfung der Atempumpe schwieriger. Sie sollte deshalb in spezialisierten Weaningzentren erfolgen. Nach der Extubation hilft eine NIV, ein Postextubationsversagen, also Reintubationen und Tracheotomien zu vermeiden. Für das weaning ist eine S2Leitlinie in Vorbereitung.

\section{NIV verbessert Langzeitüberleben}

Viele COPD Patienten behalten eine chronisch-hyperkapnische Insuffizienz infolge der mechanischen Dysfunktion, Störungen der Atemregulation und Dysfunktion der Atemmuskulatur. Blutgase, Lungenfunktion und Langzeitüberleben dieser Patienten können durch eine intermittierende häusliche NIV deutlich verbessert werden, erklärte Prof. Michael Pfeifer, Donaustauf. Die NIV über Nasen- oder Nasenmundmaske wird empfohlen, wenn alle konservativen Therapiemöglichkeiten ausgeschöpft sind.
Klassische Prognosefaktoren bei COPD wie $\mathrm{BMI}, \mathrm{FEV}_{1}$, Alter und Überblähung haben auch für Patienten unter NIV ihre Bedeutung. Zusätzlich empfahl Pfeifer, den leicht erfassbaren Basenüberschuss ( $>9 \mathrm{mmol} / \mathrm{L}$ ) zu messen, der die langfristige Kompensation der chronischen Hyperkapnie abbildet. Damit lässt sich nicht nur der Schweregrad der chronisch-ventilatorischen Insuffizienz beurteilen, sondern auch die Prognose.

\section{Schneller Flussanstieg erforderlich}

In der Beatmung kommt es auf einen schnellen Flussanstieg an, damit genügend Zeit für die Expiration bleibt. Speziell für COPD-Patienten hat die Firma Weinmann ein Beatmungsgerät (Ventimotion-2) entwickelt, das diese Anforderungen erfüllt.

$\mathrm{ab}$

Symposium „Nicht invasive Beatmung bei COPD - State oft he Art und Ausblick" anlässlich des 52. DGP-Kongresses am 7. April 2011 in Dresden (Veranstalter: Weinmann)

\section{DGP-Leitlinie zu akuter Bronchitis}

\section{Starke Empfehlung für Pflanzenkombination}

— In der Leitlinie der Deutschen Gesellschaft für Pneumologie und Beatmungsmedizin zur „Diagnostik und Therapie von erwachsenen Personen mit akutem und chronischem Husten" werden Sekretolytika zurückhaltend beurteilt: „Es gibt derzeit wenig methodisch

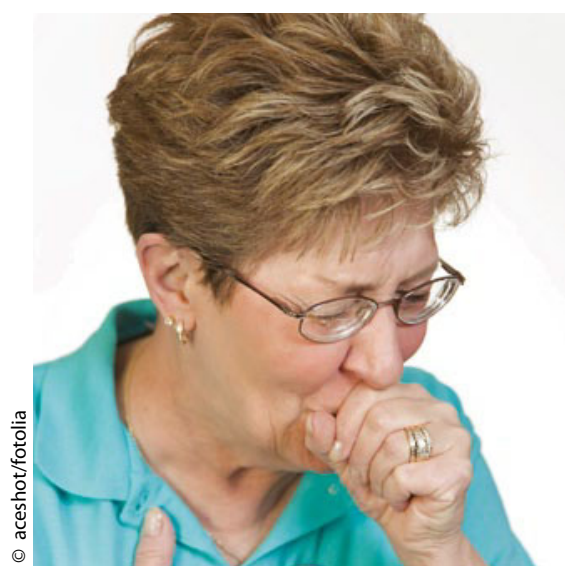

Ein pflanzliches Kombinationspräparat kann akuten Husten schnell lindern. einwandfreie Studien zur Bewertung der Wirksamkeit in Hinblick auf die Linderung des akuten Hustens", heißt es dort. Einzige Ausnahme ist das pflanzliche Kombinationspräparat Bronchipret ${ }^{\oplus}$. Für das Phytotherapeutikum wird bei „mittelgradiger Evidenz" eine „starke Empfehlung“ ausgesprochen.

Grundlage für die Empfehlung sind zwei randomisierte kontrollierte Studien, die placebokontrolliert und doppelblind durchgeführt wurden. Bronchipret ${ }^{\oplus}$ wurde in einer Studie als Saft mit Wirkstoffen aus Thymian und Efeu, in der anderen Studie in Form von Filmtabletten mit Wirkstoffen aus Thymian und Primel gegeben.

\section{$\mathbf{6 7 \%}$ weniger Hustenanfälle}

An den Studien beteiligten sich 363 bzw. 362 Patienten mit akuter Bronchitis mit produktivem Husten und erschwertem Abhusten. Die Zahl der Hustenanfälle, der primäre Studienendpunkt, war an den Tagen 7-9 mit dem Thymian-Efeu-Saft um 68,7\% (Placebo
47,6\%) zurückgegangen, mit den ThymianPrimel-Tabletten um 67,1\% (Placebo 51,3\%). Mit beiden Darreichungen konnte die Zahl der Hustenanfälle zwei Tage früher halbiert werden als mit Placebo.

\section{Sehr gute Verträglichkeit}

Die Verträglichkeit der PflanzenextraktKombination wurde von mehr als 95\% der Prüfärzte sowie der Patienten als gut oder sehr gut eingestuft.

$Z u$ vergleichbaren Ergebnissen kommt eine prospektive Anwendungsbeobachtung mit dem Thymian-Efeu-Saft bei 1234 Kindern und Jugendlichen ab zwei Jahren. Die Hustenintensität ließ schnell nach. Vier Tage nach Behandlungsbeginn war der BronchitisSymptomen-Score um die Hälfte reduziert. Auch hier wurde die Verträglichkeit als sehr gut bewertet.

red

Quellen: AWMF S3-Leitlinie Nr. 020/003 von

2010; Informationen von Bionorica SE 